\begin{tabular}{|c|c|c|}
\cline { 2 - 3 } & REVISTA SABERES APUDEP & Volumen 3 Número 2 \\
& ISSN L 2644-3805 & Julio-diciembre 2020 \\
\hline
\end{tabular}

\title{
ESTADOS FINANCIEROS: COMPETENCIA CONTABLE BÁSICA EN LA FORMACIÓN DE CONTADORES PÚBLICOS AUTORIZADOS
}

\author{
FINANCIAL STATEMENTS: BASIC ACCOUNTING COMPETENCE IN THE \\ TRAINING OF AUTHORIZED PUBLIC ACCOUNTANTS
}

\author{
Luis Sáenz \\ Universidad de Panamá, Centro Regional Universitario de Colón, \\ luis.saenz1953@gmail.com \\ https://orcid.org/0000-0001-6686-3632
}

\section{RESUMEN}

En el presente artículo se aborda, desde el punto de vista teórico y a través de los planteamientos de diferentes autores, qué son los estados financieros, cuáles son los más conocidos y quiénes son sus usuarios; como competencia básica en la formación profesional de contadores públicos en Panamá. Para el análisis documental se realizó una revisión de literatura mediante dos fases, una convergente que consistió en búsquedas en Google Académico utilizando como palabras clave: "Estados financieros y competencias contables", sin restricciones de tiempo. La fase divergente consistió en la selección de los documentos cuyos títulos y resúmenes tuvieran mayor relación con concepciones y descripciones de estados financieros en formación profesional. Esos documentos se volvieron a filtrar utilizando como criterio la utilidad dado para los usuarios. Un correcto análisis del estado financiero de la empresa orienta la toma de decisiones de la situación actual de la empresa: inversión, financiamiento, planes de acción; así como predecir el desempeño futuro de la misma.

PALABRAS CLAVE: Estados financieros, Competencia contable, formación profesional.

\begin{abstract}
This article addresses, from the theoretical point of view and through the approaches of different authors, what are the financial statements, which are the best known and who are their users; as a basic competence in the professional training of public accountants in Panama. For the design of the proposal, a literature review was carried out through two phases, a convergent one that consisted of searches in
\end{abstract}




\begin{tabular}{|c|c|c|}
\cline { 2 - 3 } & REVISTA SABERES APUDEP & Volumen 3 Número 2 \\
ISSN L 2644-3805 & Julio-diciembre 2020 \\
\hline
\end{tabular}

Google Scholar using as keywords: "Financial statements and accounting competencies", without time restrictions. Those selected documents were re-filtered using the relevance of the approaches between conceptions and the usefulness given to users as a criterion. Subsequently, the analysis of the filtered documents was carried out and coincidences between them were identified. A correct analysis of the financial state of the company guides the decision making of the current situation of the company: investment, financing, action plans; as well as predicting its future performance.

KEY WORDS: Financial statements, accounting competence, professional training

\section{INTRODUCCIÓN}

Desde mediados del siglo pasado, los países latinoamericanos, entre estos, Panamá, han impulsado su desarrollo socioeconómico tomando como base: el Comercio Internacional, la inversión extranjera directa (IED), los flujos de mercado de capitales, las normativas internacionales, códigos de ética y modelos administrativos y contables (Banco Mundial, 2000). En el desarrollo socioeconómico, caso Panamá, han participado las universidades, las empresas y el sector gubernamental, los cuales con roles diversos han aplicado sus respectivas misiones - visiones, pero sin un enfoque de conjunto, los cuales demandan valoraciones y respuestas.

Los procesos de globalización y liberación de mercados han modificado la oferta y demanda de bienes y servicios a escala mundial; los avances tecnológicos han hecho que el mundo se convierta en un sistema dinámico de cambio constante que obliga a crear e innovar incesantemente como exigencia competitiva. Este panorama ha provocado un ecosistema fundamentalmente novedoso en las relaciones históricas universidad empresas y el sector gubernamental (Fernández, 2019). 


\begin{tabular}{|c|c|c|}
\cline { 2 - 3 } & REVISTA SABERES APUDEP & Volumen 3 Número 2 \\
ISSN L 2644-3805 & Julio-diciembre 2020 \\
\hline
\end{tabular}

Por otro lado, las NIIF (Norma de Información Internacional Financiera), señalan que las empresas que radican en Panamá tienen la obligación de emitir sus estados financieros conforme a las Normas Internacionales de Información Financiera, y a su vez los auditores externos que revisen esa información financiera deben utilizar las Normas y Procedimientos de Auditoria Internacionales.

Todo ello orientado a la tendencia internacional de contar con procesos globalizados que faciliten al usuario de la información financiera contar con un conjunto único de normas aplicables en cualquier parte del mundo, que permitirán comparar de un país a otro los estados financieros de las empresas sobre una misma base de preparación y presentación (Duran Ortiz, Mejía Hernández y Reyes, 2012).

Los estados financieros proporcionan información acerca de la situación financiera, el desempeño, y los cambios que se producen en la situación financiera de una empresa que es útil para una gran variedad de usuarios de los estados financieros al momento de la toma de decisiones económicas. Se reconoce que los estados financieros no proporcionan toda la información que los usuarios pueden necesitar al momento de la toma de decisiones económicas, puesto que ellos están orientados en gran parte hacia el efecto financiero que produce la realización de eventos en el pasado (Isaac et al, 2009; Romero López, 2002; Rojas y Aguilar; 1996) Las finanzas constituyen una de las áreas pilares que permite y facilita la toma de decisiones en la administración, siendo la valoración de empresas una herramienta de vital importancia en cualquier estrategia empresarial, desarrollándose a lo largo de la historia diferentes métodos con el objetivo de encontrar una correcta valoración, lo cual requiere precisión, cuidado y permite principalmente entre otras aplicaciones tener una mayor claridad del valor financiero de la organización para los inversionistas y demás actores económicos. 


\begin{tabular}{|c|c|c|}
\cline { 2 - 3 } & REVISTA SABERES APUDEP & Volumen 3 Número 2 \\
ISSN L 2644-3805 & Julio-diciembre 2020 \\
\hline
\end{tabular}

Las finanzas, a su vez, comprenden un proceso de gestión y gerencia de cada organización indistintamente de su tamaño y del sector al que pertenezca dicha empresa. Se torna indispensable poder realizar y ejecutar el análisis, decisiones y acciones a proceso de evaluación, en donde las mismas deben estar relacionadas con la estrategia y medios financieros que sean necesarios para sostener la operatividad de la organización propiciando al mismo tiempo el margen de utilidad para los accionistas (Cabrera, Fuentes y Cerezo;2017).

La función financiera integra todas las tareas relacionadas con el logro, utilización y control de recursos. Si se logra comprender que la gestión financiera está relacionada con la toma de decisiones relativas al tamaño y composición de los activos, a nivel de estructura de la financiación y a las políticas de los dividendos, esto lleva a pensar que cada organización debe y puede definir sus objetivos estratégicos desde diferentes perspectivas, de acuerdo a sus necesidades. Dicho de otra manera, todo objetivo de la gestión financiera debe permitirles a las empresas manejarse de manera transparente y eficiente.

Es por ello que la formación de profesionales Contables, a nivel de educación superior, debe garantizar que estos egresados posean competencias básicas y específicas que le garanticen ser competente en el ejercicio de su profesión. Es decir, pueda integrarse a la tarea con los demás, en busca de un bien cooperativo (Tobón, 2010) 


\begin{tabular}{|c|c|c|}
\cline { 2 - 3 } & REVISTA SABERES APUDEP & Volumen 3 Número 2 \\
\hline
\end{tabular}

Algunos estudios señalan que la disciplina contable no es enseñada comvo una disciplina científica, sino como un saber práctico al insistir que en la formación de los contables estos o desarrollan una experticia práctica sin suelo teórico, o se transforman en eruditos, amplios conocedores de teorías sin aplicación práctica de sus saberes (Cortes, 2006; García- Jiménez, 2014).

La relación entre la disciplina contable, la formación de competencias contables y la gestión financiera como cuerpo teórico y conceptual, estructurado bajo la premisa de que el acto formador es un acto científico, intencionado y explicable a través de postulados científicos es escasa a nivel de la Región Neo tropical.

\section{ANTECEDENTES}

Entre las escasas investigaciones que tratan de referenciar el desarrollo de competencias contables, la formación contable y la gestión financiera se encuentra:

"Interpretación de los estados financieros en términos fiscales en la formación del profesional contable", tema abordado por Medellín Ramírez (2015), quien aborda en un estudio realizado en la Universidad Autónoma San Juan de Potosí, México, los Estados Financieros, su normatividad, su proceso de elaboración y la importancia de su uso como herramienta en el proceso de toma de decisiones en materia contable y fiscal, así como las obligaciones a que esto conlleva para lo cual propone utilizar para su aprendizaje el método basado en la resolución de problemas, el cual busca desarrollar en el alumno un sentido crítico para que puedan analizarlos e interpretarlos correctamente en el desempeño de su práctica profesional

García - Jiménez, (2014) quien planteo en un estudio realizado en la Universidad La Gran Colombia de Armenia, y la Universidad del Quindío, Colombia, elementos para la comprensión de la enseñanza de la contabilidad como disciplina 


\begin{tabular}{|c|c|c|}
\cline { 2 - 3 } & REVISTA SABERES APUDEP & Volumen 3 Número 2 \\
\hline
\end{tabular}

académica, problematizando el acto formativo y determinando razones para las prácticas tradicionales de enseñanza en programas de Contaduría Pública.

Ríos León, (2013) en estudio realizado en Bogotá Colombia apunta a la necesidad de fortalecer los programas académicos de Contaduría Pública en investigación para potenciar el desarrollo profesional de los estudiantes, ya que les ayuda a comprender problemas y a buscar soluciones oportunas y adecuadas tanto en su contexto como el de las organizaciones a las cuales sirven o están vinculados.

"Análisis de Estados Financieros para la mejor toma de decisiones" tema discutido por González et al (2012), Universidad Politécnica Nacional, quienes proponen una guía básica para orientar el análisis financiero de una empresa comercial mexicana tomando en cuenta los tiempos para la toma de decisiones adecuadas.

Miranda Robles, (2009) hace una revisión del uso de tecnología de información contable y propone un modelo para el desarrollo eficiente y productivo de las empresas contables en la Provincia de Trujillo, Perú.

Selter, (2001) quien realizó un compendio de experiencias docentes sobre didáctica creativa en la universidad, y más específicamente, en los programas de contabilidad, a través de entrevistas presentadas en forma de dialogo, donde participaron docentes de universidades argentinas y españolas. Este ejercicio investigativo le permitió establecer categorías de contenido o reflexiones sobre las discusiones en relación con el estatus epistemológico de la contabilidad.

Por otro lado, existen diferentes propuestas sobre formación por competencias en contaduría, elaborados tanto por entidades como la Organización para la Cooperación y Desarrollo Económico (OCDE), la Organización Internacional del Trabajo 


\begin{tabular}{|c|c|c|}
\cline { 2 - 3 } & REVISTA SABERES APUDEP & Volumen 3 Número 2 \\
\hline
\end{tabular}

(OIT), el Banco Mundial, el Instituto Mexicano de Contadores, el Colegio de Contadores de Chile, la International Federation of Accountants (IFAC) y la International Accounting Standards Board (IASB), entre otros.

Este articulo tiene como propósito presentar los posicionamientos teóricos sobre Estados Financieros, como competencia básica en la formación profesional de contadores públicos en Panamá. Para el diseño de la propuesta se realizó una revisión de literatura mediante dos fases, una convergente que consistió en búsquedas en Google Académico utilizando como palabras clave: "Estados financieros y competencias contables", sin restricciones de tiempo. En esta fase se consiguió un total de 16,500 documentos; la fase divergente consistió en la selección de los documentos cuyos títulos y resúmenes tuvieran mayor relación con concepciones y descripciones de estados financieros en formación profesional.

Esos documentos seleccionados se volvieron a filtrar utilizando como criterio la pertinencia los acercamientos entre concepciones y utilidad dado por los autores. Posteriormente se realizó el análisis de los documentos filtrados y se identificaron coincidencias entre estos.

\section{DESARROLLO}

\section{ESTADOS FINANCIEROS}

Los estados o informes financieros son documentos donde se plasman estructuradamente y de forma concreta los resultados de las operaciones o actividades realizadas por una entidad económica, su situación financiera y los cambios experimentados en esta, a una fecha determinada, con la finalidad de satisfacer las necesidades de información que tenga la administración y los terceros interesados en la empresa (Rojas y Aguilar, 1996; Romero López, 2002). Esos datos pueden ser empleados en el proceso de toma de decisiones. 


\begin{tabular}{|c|c|c|}
\cline { 2 - 3 } & REVISTA SABERES APUDEP & Volumen 3 Número 2 \\
\hline
\end{tabular}

En este aspecto, Rodríguez (2009), indica que un estado financiero es un documento primordialmente numérico que proporciona informes periódicos o a fechas determinadas, sobre el estado o desarrollo de la administración financiera de una empresa.

Para Miranda Robles (2009), los Estados Financieros son Informes cuantitativos sobre la situación financiera y los resultados de las operaciones de los participantes del mercado, que deben ser remitidos periódicamente a la Superintendencia General de Valores. Incluyen información de activos, pasivos y patrimonio, así como de ingresos y gastos.

González et al (2012), señala que los Estados Financieros constituyen las principales fuentes de información a la que recurren los diferentes usuarios de la información para saber la situación económica de una entidad.

Rueda Rodríguez y Tamayo Ibáñez (2015), hacen referencia sobre los Estados Financieros como "documentos que evidencian el proceso contable de una entidad en un periodo determinado de acuerdo a principios o normas contables vigentes".

Desde nuestro punto de vista, los registros contables y los estados financieros son componentes fundamentales en la contabilidad, puesto que la información incluido en estos documentos son reflejados por transacciones y reportes de los libros contables, permitiendo a los usuarios tomar decisiones que mejoren la solvencia, rentabilidad, y actividad de la empresa. 


\begin{tabular}{|c|c|c|}
\cline { 2 - 3 } & REVISTA SABERES APUDEP & Volumen 3 Número 2 \\
\hline
\end{tabular}

\section{TIPOS DE ESTADOS FINANCIEROS}

Medellín Ramírez (2015), establece que hay muchos estados financieros o informes que cada empresa podrá utilizar de acuerdo a sus necesidades específicas de información, sin embargo, las Normas de Información Financiera prevén solo cuatro de ellos como estados financieros básicos que son: Estado de Situación Financiera Estado de Resultado Integral, Estado de Cambios en el Capital Contable y Estado de Flujos de Efectivo.

\section{A. Estado de Situación Financiera o Balance General}

El Estado de Situación Financiera o Balance General es comúnmente definido como el documento que presenta la situación financiera de un ente económico a una fecha determinada expresada en términos monetarios. En él se presenta detalladamente los recursos que posee la empresa, las obligaciones que tiene con sus acreedores y la participación que tienen los dueños o accionistas en ella. Este documento permite, a través de su análisis, conocer la solvencia, la liquidez y la eficiencia operativa del ente que lo emite. Para presentar esta información, se clasifica y agrupa en tres categorías o grupos principales: activos, pasivos y patrimonio o capital contable.

La Norma de Información Financiera B-6 (NIF B-6) permite el uso indistinto de tres denominaciones para este estado financiero: Estado de Situación Financiera, Balance General y Estado de Posición Financiera (Moreno Fernández, 2002; Leal González, 1999; NAFINSA, 2004) 


\begin{tabular}{|c|c|c|}
\cline { 2 - 3 } & REVISTA SABERES APUDEP & Volumen 3 Número 2 \\
\hline
\end{tabular}

Elementos del Informe de Balance General

El Balance General se debe elaborar "con los saldos de las cuentas del activo, pasivo y capital contable que se obtengan al finalizar el período contable o ejercicio" (Romero López, 2002).

En cuanto a la forma, este documento debe estar integrado por tres componentes: Encabezado, con la indicación del nombre, razón o denominación social de la entidad, la designación de Balance General y la fecha a la cual se formula; el cuerpo, conformado por las cuentas de activo, pasivo y capital contable correctamente presentadas y el pie, donde se colocan las firmas de las personas que intervienen en su elaboración y autorización; además, cuando corresponda, la leyenda de que las notas que se acompañan son parte del estado (Romero López, 2002)

\section{Activo}

El activo es comúnmente identificado como todos aquellos recursos monetarios, en especie y la parte diferida de cualquier costo o gasto incurrido que posee una entidad económica a una fecha determinada para hacer frente a sus obligaciones y mantener en marcha sus operaciones normales (Rojas y Aguilar, 1996). Se clasificará el activo como activo a corto plazo o circulante y activo a largo plazo o no circulante. Estas denominaciones se pueden utilizar de forma indistinta según lo establecido en las bases para conclusiones BC6 de la NIF B-6. El activo a corto plazo se define como aquellos recursos e inversiones con los que cuenta la entidad que se harán efectivos en un plazo no mayor a un año y que servirán para pagar a los acreedores en ese mismo lapso de tiempo y mantener a la empresa en funcionamiento. 


\begin{tabular}{|c|c|c|}
\cline { 2 - 3 } & REVISTA SABERES APUDEP & Volumen 3 Número 2 \\
\hline
\end{tabular}

Por su parte, el activo no circulante será aquel que transformarán sus conceptos en efectivo en un plazo mayor de un año y se puede emplear en pagar deuda que contraiga la empresa también a largo plazo (Prieto, 2009)

Pasivo

El pasivo es el total de deudas y obligaciones contraídas por un ente económico por concepto de compra de mercancías o servicios adquiridos a crédito, salarios y sueldos por pagar a los empleados, impuestos, documentos e hipotecas por pagar entre otros, por los cuales la empresa se obliga a pagar a una fecha determinada con bienes, dinero o servicios (Rojas y Aguilar, 1996).

Capital Contable

El capital de un negocio representa el interés de los propietarios en la sociedad, dividido en acciones o unidades, por lo que indicará la porción de los activos que se están financiando con los recursos de estos (Pyle, White, y Larson, 1981).

Balance General consolidado

Un Balance General consolidado es un estado financiero que muestra en forma combinada los activos, pasivos, ingresos, gastos y flujos de efectivo de un grupo de empresas, como si fuesen una sola y busca mostrar adecuadamente los riesgos a los que están expuestos los inversores en estas entidades. Este grupo de empresas se divide en una empresa matriz y varias subsidiarias que están siendo operadas bajo un control común o unificado por parte de la matriz como si fuesen un solo ente económico, aunque ante la ley cada una pueda tener derechos y obligaciones distintas (García Mendoza, 1998). 


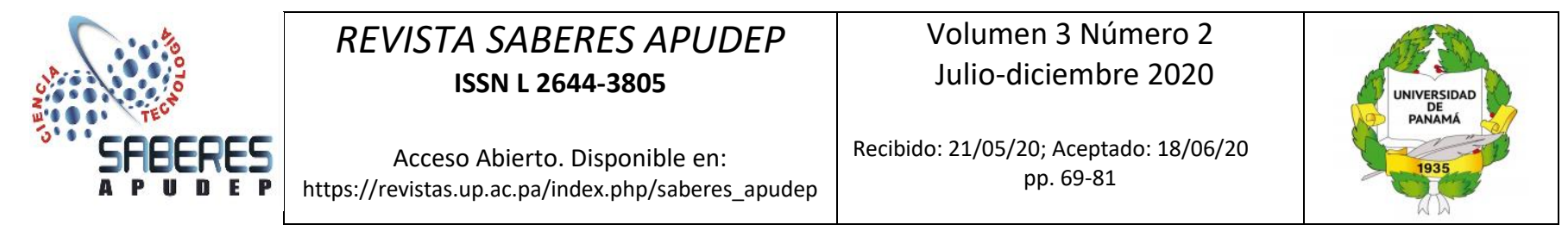

\section{B. Estado de Resultado Integral}

El Estado de Resultado Integral o de pérdidas y ganancias es el documento financiero que muestra la diferencia entre el monto de los ingresos y de los gastos de una entidad económica por un período determinado, la cual será denominada utilidad o pérdida según corresponda y servirá para que los usuarios de la información financiera estén en posibilidad de evaluar los cambios potenciales en los recursos económicos futuros, para predecir el potencial de la entidad para generar flujos de efectivo y para estimar la eficiencia y la eficacia con que se pueden utilizar estos recursos y con esto tomar las decisiones operativas pertinentes para mejorar la rentabilidad del negocio.

La utilidad o pérdida obtenida modifica el capital contable del ente económico en el Estado de Situación Financiera (Guajardo Cantú y Andrade de Guajardo, 2008).

\section{Estado de Cambios en el Capital Contable}

Morán Mendoza (2013), señala que el Estado de Cambios en el Capital Contable es el estado financiero que refleja las modificaciones sufridas por los elementos del capital contable de una entidad durante un período contable determinado, derivado de las decisiones tomadas por sus propietarios en relación con su inversión en dicha entidad, motivo por el cual es posible evaluar el comportamiento de la administración en el logro de sus objetivos ya que también se presentan tanto los resultados del citado período como de ejercicios anteriores y los movimientos efectuados en estas cuentas de capital. El Estado de Cambios en el Capital Contable también evalúa los cambios experimentados en el capital contable derivados de operaciones como capitalizaciones, aportaciones, distribuciones y reembolsos que hayan ocurrido en el período que comprende el estado financiero. 


\section{REVISTA SABERES APUDEP \\ ISSN L 2644-3805}

Acceso Abierto. Disponible en:

https://revistas.up.ac.pa/index.php/saberes_apudep
Volumen 3 Número 2

Julio-diciembre 2020

Recibido: 21/05/20; Aceptado: 18/06/20 pp. $69-81$

\section{Estado de Flujos de Efectivo}

El Estado de Flujos de Efectivo tiene como objetivo principal proporcionar información acerca de los ingresos y pagos de efectivo, actividades de operación, inversión y financiación de la entidad al informar a los usuarios acerca de la procedencia de los fondos que la empresa ha utilizado durante un período determinado, así como del uso que se les ha dado durante este tiempo para que con esto la empresa tenga herramientas suficientes para realizar diagnósticos relacionados con la capacidad que tiene la entidad de obtener financiamiento externo y determinar si la empresa está creciendo con recursos generados por sí misma o por motivo de estos montos obtenidos de terceros. Además, conocerá su capacidad para generar flujos con efectivos positivos.

Con la información generada en este estado financiero, los usuarios podrán evaluar la capacidad de la empresa para cumplir con sus obligaciones con terceros y la retribución al capital la cual se reflejará en el pago de dividendos a los socios.

Para informar lo anterior, este estado financiero divide los movimientos de efectivo de la empresa y sus equivalentes en actividades operativas, de inversión y de financiamiento y la relación entre estas tres secciones dará como resultado el flujo del negocio (Rosas Inostroza, 2009).

\section{USUARIOS DE LOS ESTADOS FINANCIEROS}

Muñoz Merchante (2009). Indica que suelen distinguirse dos grandes grupos de usuarios de los Estados Financieros: los usuarios externos, que carecen de atribuciones directas con respecto a la gestión empresarial y los usuarios internos o responsables de la gestión. 


\begin{tabular}{|c|c|c|}
\cline { 2 - 3 } & REVISTA SABERES APUDEP & Volumen 3 Número 2 \\
\hline
\end{tabular}

\section{A- Usuarios Externos}

1. Hacienda Pública. Gran parte de los impuestos que los administradores públicos recaudan procede de las empresas y han de ser reconocidas por estas en sus registros contables, para informar así sobre el cálculo, control y revisión de los mismos.

2. Entidades Bancarias. Además de prestar numerosos servicios a las empresas, suelen ser sus principales financiadores o prestamistas externos. Por ello necesitan conocer la información financiera relativa a las empresas prestatarias para determinar si estas serán capaces de devolver a su vencimiento los compromisos adquiridos, tanto por el capital prestado como los intereses estipulados, o para decidir sobre las solicitudes de nuevos préstamos.

3. Proveedores y otros acreedores. Aquí se engloban a los acreedores no financieros esto es los suministradores de bienes y servicios adquiridos por la empresa a menudo con pago aplazado. Por lo que su principal necesidad será disponer de la información que les permita conocer la capacidad de la empresa para atender sus pagos a corto y largo plazo

4. Clientes. Son los adquirientes habituales de los bienes y servicios producidos por la empresa y demandaran información a esta con el fin esencial de evaluar su continuidad, especialmente cuando su actividad dependa de manera importante de tales adquisiciones.

5. Otros usuarios. Entre los potenciales usuarios de la información financiera sin relación contractual con la empresa, figuran los organismos públicos, encargados de regular determinados aspectos de la actividad económica como son: Sindicatos y organizaciones empresariales por su papel de mediadores en el mercado de trabajo, las empresas competidoras y analistas financieros. 


\begin{tabular}{|c|c|c|}
\cline { 2 - 3 } & REVISTA SABERES APUDEP & Volumen 3 Número 2 \\
\hline
\end{tabular}

B - Usuarios internos

1. Socios y Accionistas. Precisarán información, esencialmente, para decidir sobre la conveniencia de mantener, reducir o incrementar su participación.

2. Empleados. Sus necesidades de información estarán directamente relacionadas son sus preocupaciones respecto a la empresa de la que dependen: perspectivas económicas, continuidad del negocio o mejora de sus retribuciones.

3. Directivos y gestores de la empresa. Cada uno en su respectiva área de responsabilidad, demandarán información financiera.

4. Área de producción. será necesario decidir que bienes producir; en el área financiera, qué recursos se precisan y como obtenerlos.

5. Área comercial. Demandará información que permita fijar el precio de venta de los bienes y servicios producido

\section{CONCLUSIÓN}

El Conocimiento de los Estados Financieros, competencia básica en la formación de profesionales contables, constituyen una herramienta fundamental, que va a permitir determinar la situación actual de la empresa y predecir el desempeño futuro; refleja el manejo de los recursos financieros disponibles, para el proceso productivo, la cual lleva implícito la detención de las deficiencias y desviaciones ocurridas, durante la gestión empresarial.

\section{REFERENCIAS BIBLIOGRAFICAS}

Banco Mundial. (2000). PREM. Grupo de políticas económicas y Grupo de economía para el desarrollo. Disponible en

http://www.bancomundial.org/temas/globalizacion/cuestiones1.htm

Cortés, J. (2006). El pensamiento crítico: Algunas reflexiones en torno a la educación contable. En: Cuarto Foro de Educación contable. 


\begin{tabular}{|c|c|c|}
\cline { 2 - 3 } & REVISTA SABERES APUDEP & Volumen 3 Número 2 \\
\hline
\end{tabular}

Durán Ortiz, M., Mejía Hernández, M y Arturo Reyes, A. (2012). Competencias genéricas y profesionales: un enfoque analítico de la licenciatura en Contaduría. XVII Congreso Internacional de Contaduría Administración e Información. Recuperado de http://premio.investiga.fca.unam.mx/docs/ponencias/2012/8.1.pdf

Fernández, O. (2019). Plataforma Científico Tecnológica Y Social Para Un Modelo Estratégico Innovador De Vínculo Universidad-Empresa- Estado. En Prensa

García - Jiménez, M. (2014). Enseñanza de la Contabilidad como disciplina académica. Concepciones de ciencias del profesorado y pensamiento crítico. Entramado 10 (1): 164.174.

García Mendoza, A. (1998). Principios de contabilidad - Análisis de los boletines series A-D. México: Mc Graw Hill

González, I; Martínez, S; Moncada, A; Santamaría, N y Vásquez, R. (2012). Análisis de Estados Financieros para la mejor toma de Decisiones. Tesis de Grado. Instituto Politécnico Nacional. México.

Guajardo Cantú, G., \& Andrade de Guajardo, N. E. (2008). Contabilidad financiera. México: McGraw Hill/Interamericana editores SA de CV

Isaac, J; Flores, O; Hernández, F; Loredo Torres, C y Isaac García, C. (2009). La rentabilidad económica (ROA) en las PYMES exportadoras de Monterrey, Nuevo León México. Observatorio de la Economía Latinoamericana. 116. Recuperado http://www.eumed.net/cursecon/ecolat/mx/2009/ifhtg.htm

Leal González, R. (diciembre de 1999). Apuntes de análisis e interpretación de los estados financieros. Tesis de licenciatura. Monterrey, Nuevo León, México: Universidad Autónoma de Nuevo León.

Medellín Ramírez, J. (2015). Interpretación adecuada de los estados financieros en términos fiscales en la formación del profesional contable. Tesis de Maestría. Universidad Autónoma de San Luis Potosí. México.

Miranda Robles, J. (2009). El uso de las tecnologías de la Información y sus implicaciones en el desarrollo contable moderno en empresas comerciales de la Provincia de Trujillo. Tesis Doctoral. Universidad Nacional de Trujillo. Perú.

Morán Mendoza, E. M. (2013). Guía completa para el examen de cene val de contadores. San Luis Potosí, México: J. Benito Posadas García.

Moreno Fernández, J. (2002). Contabilidad superior. México: Instituto Mexicano de Contadores Públicos AC.

Muñoz Merchante, A. (2009). Análisis de Estados financieros. Teoría y práctica. (2ª ed.). Madrid: Ediciones Académicas 


\begin{tabular}{|c|c|c|}
\cline { 2 - 3 } & REVISTA SABERES APUDEP & Volumen 3 Número 2 \\
\hline
\end{tabular}

NAFINSA. (2004). Fundamentos de negocio. Recuperado file://C:/Users/Emilio/Documents/Cayune\%20SA\%20de\%20CV/CONTABILIDAD \% 202014/administracion1_2.pdf

NIIF. (2016). Guía rápida de las NIIF Normas de Información Financiera.

https://www2.deloitte.com/content/dam/Deloitte/pa/Documents/audit/Deloitte-ES-

Auditoria-guia- rapida-NIIF-2016.pdf

Prieto, A. (2009). Contabilidad básica enfocada a Normas de Información Financiera.

México: Editorial Limusa SA de CV.

Pyle, W. W., White, J. A., \& Larson, K. D. (1981). Principios fundamentales de contabilidad. México: Compañía editorial continental SA.

Ríos León, R. (2013). La formación para la Investigación de Contadores Públicos Colombianos: un asunto de estrategias. Revista Científica General José María Córdova Bogotá. 11 (11): 175-206

Rojas y Aguilar, H. (1996). Apuntes de análisis e interpretación de los estados financieros. México: Instituto Politécnico Nacional

Selter, J. (2001). La aplicación de una didáctica creativa en la enseñanza de contabilidad. Revista Fuentes. 3: 87-106c

Tobón Tobón, S. (2010). Formación Integral y Competencias. Pensamiento Complejo, currículo, didáctica y evaluación. 3Ed. Centro de Investigación en Formación y Evaluación. CIFE, Bogotá, Colombia Ecoe Ediciones. 